\title{
Study on VFTO of XiMeng UHV substation 1000kV GIS substation
}

\author{
Xiu-Cheng Su,,Bo-Han Liu†, Li Bo, Guo-Zeng Yan, Xiang-Ping Ni and Zhen-Tao Liu, \\ State Grid AC Engineering Construction Company, Xicheng District, Beijing 102206, \\ China \\ ${ }^{\dagger}$ E-mail:ncmomo@163.com
}

\begin{abstract}
This paper presents the VFTO calculation and analyzing results of XiMeng UHV pilot project in China. The result shows that the maximum voltage amplitude of VFTO in GIS is $1981 \mathrm{kV}$, so that the pre-insert switching on off resistance is not necessary to installed on disconnector. In this case, it will reduce the probability of GIS failure operation and the potential supply current, and the safety and reliability of UHV GIS system will be improved. Meanwhile,the VFTO of a transformer in GIS substation is not high when the transformer is connected to GIS by overhead line.
\end{abstract}

Keywords: UHV Substation; Transmission; GIS; VFTO; Insulator.

\section{The Characteristics and Causes of VFTO}

As a result of SF6 insulation fully enclosed type combined electric has the characteristics of small size, using small area, reliable operation, easy maintenance and so on, it has been used widely. GIS isolation switch in the off load short bus, will produce rekindling many times, the voltage at both ends of the contact gap in a few nanoseconds suddenly rise or drop, the voltage wave produced the traveling wave propagated in the GIS, when the wave impedance changing will produce the refraction reflection, the multiple refraction and reflection of GIS in various traveling wave component will added together, the formation of fast transient overvoltage (VFTO) will produced. The initial traveling wave rise time and amplitude have certain influence on the final form of the very fast transient overvoltage waveform, and the amplitude is determined by the actual conditions of isolation switch breakdown moment at both ends of each side of the bus, such as the length, the position of impedance change. These will be followed by the decision of the whole transient over voltage waveform of the peak [1-3].

SF6 gas filling in GIS itself has very high dielectric strength, internal electric field formed mostly close to uniform electric field, electric strength in a uniform electric field of SF6 is about 3 times the air gap distance, so does the same voltage level of the small air gap, the gap between the breakdown process 
is very fast. When the switch operation, the electric field between the contacts as a department of uniform electric field, SF6 contact gap breakdown, discharge speed, injection network with high step wave steepness, rise time of VFTO normally for several ns to dozens of ns. So generally only in the GIS device VFTO problems exist. The circuit breaker in GIS is also likely to produce VFTO, but because of their speed was very fast, so it rarely happens again. While the disconnector switching speed is low, in the off operation process, will produce dozens of times again, so the VFTO problem in GIS is mainly composed of isolating switch operation.

\section{The Damage of VFTO for the Sysytem}

Very fast transient overvoltage (VFTO) effects the different parts in GIS (such as the isolation switch itself, insulation gap and insulator and bushing), they may cause the failure, and it will cause great danger for the other nearby high voltage equipment (such as power transformer insulation structure) [1].

The operation process of isolating switch, the contacts moved slowly moving, will cause many pre breakdown or a contact between the wear process of closing contact occurs near the pre breakdown two. As the operating speed is slow. For the first time, it is necessary to occur when the power frequency voltage peak. The breakdown current is charged to the power supply voltage, and the potential difference between the contacts is decreased, the spark discharge goes out, and the residual charge is left in the short term. Subsequent breakdown is similar to the coincidence gate, which can lead to a higher voltage magnitude of VFTO. In the process of opening, also due to the moving speed of the moving contact is slow, the two contact will occur between the critical wear, also similar to the same, can also lead to higher VFTO value.

For the new construction of Ximeng - Shandong UHV AC power system $1000 \mathrm{kV}$ Ximeng UHV substation, this problem is more prominent, because the higher the system rated voltage, lightning impulse withstand voltage (LIWV) equipment and system rated voltage is lower than Un. Compared with the rated voltage of $1000 \mathrm{kVGIS}$ substation and 500kVGIS substation, the relative value of VFTO is basically the same, and the absolute value of VFTO is increased by 1 times compared with the rated voltage. But the insulation level of $1000 \mathrm{kVGIS}$ equipment (LIWV) only increased by 55\% compared to 500kVGIS, there is no proportion increase, so the VFTO of the ultra-high pressure GIS is greater than the harm of 500kVGIS.

At present, the IEC insulation coordination standard has not yet determined the typical waveform of the VFTO test. Generally considered for the GIS device, VFTO can be compared with LIWV. GIS LIWV 2400kV, can be considered a safety margin of $15 \%$ VFTO, not more than $2087 \mathrm{kV}$. For GIS devices, IEC 
IEC60071-2:1996[5-8] believes that the insulation insulation with the standard safety margin should be larger, $20 \%$. Considering the VFTO wave front time is shorter than the lightning impulse wave time, the voltage of GIS insulation is slightly increased. The comparison between VFTO and LIWV when the safety margin of $15 \%$ is appropriate.

VFTO wavefront is very steep, the voltage distribution in transformer winding pole uniform, introduced in the initial part of the ultra high voltage winding will produce high voltage drop, easy to cause the interturn insulation damage for VFTO main transformer, the harm of interturn insulation. The distribution and size of VFTO in turn insulation are related to the VFTO frequency and the structure of the inner winding of the transformer. At home and abroad have occurred VFTO damaged GIS $>420 \mathrm{kV}$ equipment and transformers and two equipment accident [1,3].

Because the Ximeng 1000kV substation is through overhead line and GIS casing is connected with the middle distance, the VFTO attenuation, does not pose a threat to the transformer. The focus of this study is the harm of VFTO to GIS ontology.

\section{The Simulation of VFTO}

VFTO's research methods include theoretical analysis, numerical simulation and field test. Methods of laboratory and field tests to get close to the VFTO characteristics in the actual operation, but due to the influence factors of the VFTO complex, more practical operation mode of substation, only through the test difficult to study VFTO features a large number of operating modes at the same time, the test is restricted by measuring means and methods: digital simulation is relatively easy to implement, VFTO research able to do a lot of the current forecast for VFTO substation also rely mainly on in digital simulation.

This study assumes that the load side of the isolation switch is 1p.u., and the power supply side voltage is -1p.u., which is the most serious VFTO.

\subsection{Simulation model of the main components}

VFTO simulation calculation, the establishment of GIS element simulation model and the selection of parameters determine the accuracy of the simulation, this study uses a more general VFTO simulation model, the main components of the simulation model is as follows:

(1) GIS pipeline

In general, the distributed transmission line model is adopted, and the wave impedance can be calculated by the Eq.(1).

$$
Z=60 \ln (R / r)
$$


$\mathrm{R}$ is the inner radius of the GIS pipe shell, and $\mathrm{R}$ is the outer radius of the inner conductor of the GIS pipeline.

In this project, GIS pipeline wave impedance is $75.2 \Omega$.

(2) Isolation switch

For the disconnection of the isolation switch generally use the segment transmission line, fracture surface capacitance and the ground capacitance, this study used the model as Figure 1 shown. For the closure of the isolation switch side to ignore its fracture capacitor, according to the information provided, $\mathrm{C} 1$ is $65 \mathrm{~F}, \mathrm{C} 2$ is $50 \mathrm{~F}, \mathrm{C} 12 \mathrm{~F}$.

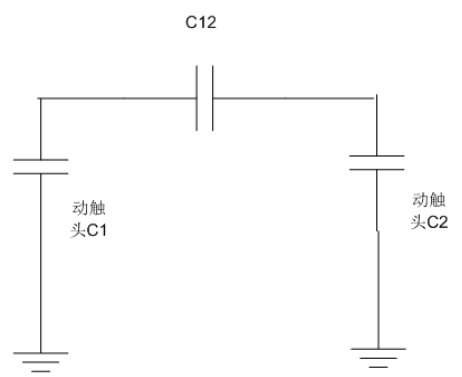

Fig.1 The simulation model of Isolating switch

(3)Circuit Breaker

In general, the fracture surface of the circuit breaker is pressed by the fracture surface and the capacitance of the ground capacitance is expressed, and the transmission characteristics are simulated by the distributed facsimile transmission line model. This study uses the model as shown in Figure 2. A closed circuit breaker, generally as part of a transmission line. The distributed parameters are used to represent and ignore the average voltage of the capacitor. According to the information provided, C1-C5 are 50uF, $\mathrm{C} 12$ and $\mathrm{C} 45$ are 2700uF, C23 and C34 are 2500uF.

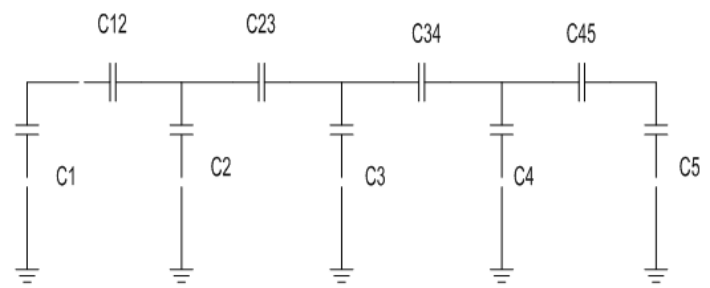

Fig.2. The simulation model of Circuit Breaker

(4)Lightning arrester, voltage transformer, insulator are used to express the ground capacitance.

According to the information provided, the main equipment parameters of the capacitor as shown in Table 1. 
Table 1. The ground capacitance of the main equipment

\begin{tabular}{ll}
\hline Equipments & Capacitance $(\mu \mathrm{F})$ \\
\hline PT of GIS bus & 200 \\
Bushing & 410 \\
Basin Insulator & 10 \\
Transformer & 600 \\
UHV reactors & 400 \\
\hline
\end{tabular}

\section{The Simulation Results of Isolation Switch}

The main wiring diagram is shown in Figure 3.

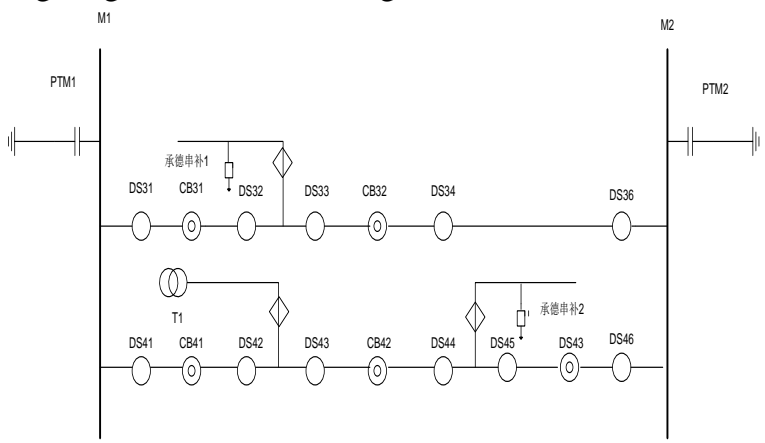

Fig.3. The configuration of the Ximeng station

GIS isolation switch operation is generally between opening and closing switch to a circuit breaker for short-term overvoltage generally appear in the short term, if the empty bus switch power source side is connected with the operation of appropriate length, in the empty bus end because of the multiple wave catadioptric may occur with high voltage. The maximum over voltage may appear at the end of the empty bus. GIS isolation switch operating frequency high voltage (hundreds of $\mathrm{kHz}$ to ten $\mathrm{MHz}$ ), the frequency depends on the high short-term end to the power supply side circuit recent negative reflection point (such as CVT, transformer, capacitance to ground the equipment in large distance). In fact, the GIS isolation switch operating over voltage waveform is formed by a variety of frequency.

The simulation calculation for each switch selects the engineering debugging or running in a typical charged operation, isolation switch operation VFTO calculation, Ximeng station the isolation switch is representative of the charged mode of operation and the isulatora switch simulation result are shown in Table 2. 
Table 2 The operation procedure of the isolating switches

\begin{tabular}{lllcl}
\hline No. & Insulator & & VFTO $(\mathrm{kV})$ \\
& Breaker & Phase A & Phase B & Phase C \\
1 & DS31 & 1800 & 1789 & 1759 \\
2 & DS32 & 1490 & 1498 & 1759 \\
3 & DS33 & 1884 & 1599 & 1583 \\
4 & DS34 & 1589 & 1719 & 1722 \\
5 & DS41 & 1808 & 1639 & 1666 \\
6 & DS42 & 1836 & 1981 & 1939 \\
7 & DS43 & 1889 & 1942 & 1852 \\
8 & DS44 & 1704 & 1842 & 1989 \\
9 & DS45 & 1793 & 1774 & 1773 \\
10 & DS46 & 1547 & 1691 & 1679 \\
\hline
\end{tabular}

From Table 3, it is clear shows that the calculation results from the isolation switch of $1000 \mathrm{kV}$ Ximeng UHV substation is not installed damping resistance, when the isolation switch operation, GIS internal VFTO peak is $1981 \mathrm{kV}$, and the lightning insulation level is $2400 \mathrm{kV}$ with the margin of $21 \%$, to meet the insulation safety margin requirements, the maximum VFTO is in the operation of DS42 isolating switc. The power operation methods are: T1 charged, remaining circuit breaker and isolating switch sides are disconnected. VFTO maximum value is $1981 \mathrm{kV}$ which appears in the CB41 circuit breaker.

\section{Conclusion}

If the maximum amplitude of isolation switch on GIS operating voltage (VFTO) can reach $2795 \mathrm{kV}$ (3.11p.u.), much more than the allowable value, the equipment insulation will be damaged in the UHV project.Therefore, adopting parallel in isolation switch in switching resistor will be used, the VFTO by the free oscillation without attenuation variable damping oscillation, reduced VFTO amplitude, speed up the voltage decay rate, but because of the advantages of compact structure of GIS, switching resistor isolating switch structure is complex, and bring potential for current increases, the problem of the single phase to ground flashover arcing time long will increase the difficulty and investment of equipment of the technique and technology in the system, thus increasing the probability of failure. In the simulation of VFTO for $1000 \mathrm{kV}$ Ximeng UHV substation project, when the switch operation, internal GIS VFTO peak is $1981 \mathrm{kV}$, and the lightning insulation level is $2400 \mathrm{kV}$ with the margin of $21 \%$, it meets the insulation safety margin requirements, so there is no need to add the shunt resistance in isolation switch, which can not only reduce the equipment and technology cost, meanwhile, it will reduce the difficulty of 
technics, and save the arc current and fault probability, the reliability of the system will be increased.

\section{References}

1. IEC 60071-1:1993 INternational standard: insulation coordinationpart1:definitions,principes and rules[S],1993.

2. IEC 60071-4:2004 INternational standard: insulation coordinationpar4:computational guide to insulation coordination and modeling of electrical networks[S],2004.

3. GB 311.1-1997 High Voltage Tansmission[S],1997.

4. IEC 60071-2:1996 INternational standard: insulation coordinationpart2:application guide[S],1996.

5. Yamagata Y. Suppression of VFT in 1100kVGIS by adopting resistor-fitted disconnector[J].IEEE Transaction on Power Delivery,1996,11(2):872880.1]P.X. Deligne and B.H. Gross, On the exceptional series, and its descendants, C. R. Math. Acad. Sci. Paris335,877 (2002). 